DOI: $10.1002 /$ adem.

\title{
Novel antibacterial and bioactive silicate glass nanoparticles for biomedical applications**
}

By Ana Catarina Vale, Ana Luísa Carvalho, Ana Margarida Barbosa, Egídio Torrado, João F. Mano, Natália M. Alves*

[*] Prof. Dr. N. M. Alves, Prof. Dr. J. F. Mano, Dr. A. C. Vale, A. L. Carvalho $3 B$ 's Research Group - Biomaterials, Biodegradables and Biomimetics, University of Minho, Headquarters of the European Institute of Excellence on Tissue Engineering and Regenerative Medicine. AvePark 4805-017 Barco, Guimarães, Portugal

E-mail:nalves@dep.uminho.pt

A. M. Barbosa, Dr. E. Torrado

Life and Health Sciences Research Institute (ICVS), School of Health Sciences, University of Minho 4701-057 Braga, Portugal

[**] The authors want to acknowledge the financial support from the Portuguese Foundation for Science and Technology through the project BioSeaGlue with the reference EXPL/CTM-BIO/0646/2013, and also the European program FEDER/COMPETE for the financial support through project LA ICVS/3Bs-2014-2015. ACV thanks Dr. Edith Ariza Avila and Mara Teixeira (SEMAT/UM) for their valuable contribution in SEM and XRD analysis, respectively.

In this work, we propose a new quick sol-gel procedure for bioglass nanoparticles production containing $10 \%$ mol of silver (AgBGs). These new AgBGs were characterized by Zeta potential analysis, scanning electron microscopy with X-ray microanalysis (SEM/EDS), X-ray powder diffraction (XRD), Fourier transform infrared spectroscopy (FTIR) and microbiological tests to confirm their bioactive and antibacterial properties. SEM showed that the average particle size was less than $200 \mathrm{~nm}$ and EDS confirmed the successful incorporation of $\mathrm{Ag} 2 \mathrm{O}$ in the bioglass matrix. XRD confirmed the amorphous nature of the AgBGs and, after SBF immersion, revealed their bioactive behavior with the presence of crystalline phase of calcium silicate and phosphorus oxide, which were also detected by FTIR analysis. FTIR also confirmed the formation of typical siloxane bonds resulting from the condensation of silicate glass. Lastly, it is found that the developed AgBGs had an 
antibacterial effect against two different types of bacteria, thus demonstrating their ability to reduce the bacterial infection within $16 h$.

\section{Introduction}

Due to their size and easy dispersability, nanoparticles can be applied in a multitude of already existing biomedical devices and strategies, enhancing mechanical properties and providing the bioactive character to a specific material, such as in the case of bioglass nanoparticles. ${ }^{[1,2]}$ Nanoparticles may also work as independent nanodevices in applications, such as nanocarriers. In particular, when bioglasses were discovered, they radically changed the biomaterials field. For the first time, a chemical bond between implants and host tissue was possible, leaving behind a first generation of bioinert devices. This was the beginning of a second generation of bioactive materials capable of inducing a cellular response at their surface. Effectively, these materials were able to bond not only to bone, but to soft tissue as well, avoiding fibrous encapsulation. ${ }^{[1,3,4]}$

It is also known that a common reason of failure in tissue integration by a biomaterial is the development of bacterial infection. In fact, the implantation of materials for prostheses and tissue regeneration is often associated with the administration of antibiotics. However, some disadvantages are connected with this prophylaxis, as allergic reactions, microbial flora depletion and bacterial resistance. ${ }^{[5]}$ Ideally, the implant should have the ability to both regenerate bone tissue and to treat the infection by delivering an antibacterial agent in a controlled and continuous manner. For these reasons, many authors have recently proposed the modification of biomaterials with different antibacterial agents, but the silver has been suggested as one of the most promising antibacterial agent. ${ }^{[6-13]}$

Silver has been known and used for centuries as one of the most interesting antibacterial material, due to its excellent broad-spectrum antimicrobial properties. ${ }^{[9,14]}$ The bacteriostatic action of silver remains unclear, but it has been suggested that silver ions kill microorganisms 
instantaneously by blocking their respiratory enzyme systems, while having no negative effect on human cells. ${ }^{[8,12,15]}$

Regarding the production of silver doped bioactive nanoparticles in the system $\mathrm{SiO}_{2}-\mathrm{CaO}-\mathrm{P}_{2} \mathrm{O}_{5}-\mathrm{Ag}_{2} \mathrm{O}$ through the sol-gel methodology, some interesting works have been already developed with different silver contents. ${ }^{[5,14,16-18]}$ In particular, Bellantone et al. ${ }^{[16]}$ developed a formulation with a $3 \mathrm{Wt}(\%) \mathrm{Ag}_{2} \mathrm{O}$, Delben et al. ${ }^{[17]}$ studied different formulations with a maximum of $5 \mathrm{~mol} \% \mathrm{Ag}$, but only El-Kady et al. ${ }^{[5]}$ and Vulpoi et al. ${ }^{[18]}$ synthesized nanoparticles with higher silver content (a maximum of $10 \mathrm{~mol} \%$ ). Attending that the addition of a medium silver content was not commonly considered, in the present work our interest was focused on the development of an alternative and simple sol-gel methodology for the synthesis of new silicate glass nanoparticles with $10 \mathrm{~mol} \%$ of silver oxide, using the same bioglass precursors described in a previous protocol developed and optimized by our group for a ternary bioglass system. ${ }^{[19]}$ Therefore, the main objective of the present study was to develop silver doped bioglass nanoparticles, which present the in vitro bioactivity already evidenced by our ternary system ${ }^{[19,20]}$ and, simultaneously, demonstrate an antimicrobial behavior to avoid severe bone infections. The produced AgBGs were, then, characterized in order to confirm the successful addition of silver oxide onto the bioglass system, and also to attest their bioactive and antibacterial properties. Several characterization techniques were performed: Zeta potential analysis, scanning electron microscopy with X-ray microanalysis (SEM/EDS), X-ray powder diffraction (XRD), Fourier transform infrared spectroscopy (FTIR); and finally, their antibacterial effect was studied through the disk diffusion methodology, against two types of bacteria with distinct Gram stains related with the respective composition of their cell walls, namely Staphylococcus aureus (Gram-positive) and Escherichia coli (Gram-negative).

\section{Experimental Section}




\subsection{Materials}

Tetraethyl orthosilicate (TEOS) $99.90 \%$ was purchased by Merck Chemicals, nitric acid monohydrate $69 \%$ was obtained by VWR. Ammonium phosphate dibasic, calcium nitrate tetra hydrate $99 \%$, ethanol absolute, ammonia water (ammonium hydrogen phosphate 98\%, maximum of $\left.33 \% \mathrm{NH}_{3}\right)$, and hydrochloric acid $(\mathrm{HCl})$ were acquired from Sigma. Silver Nitrate $\left(\mathrm{AgNO}_{3}\right)$ was obtained by VWR. The ternary bioglass nanoparticles (BGs) used in this study were produced using the method developed and optimized in our research group. ${ }^{[19,20]}$ For microbial tests, Mueller-Hinton agar (MHA) medium was purchased from ThermoScientific (Thebarton, SA, Australia) and standardized cultures of Staphylococcus aureus (ATCC 29213TM) and Escherichia coli (ATCC 25922TM) with 1.5×10 ${ }^{8}$ CFU were purchased from the American Type Culture Collection (Manassas, VA, USA).

\subsection{Methods}

\subsubsection{Synthesis of silver doped bioglass nanoparticles (AgBGs)}

Silver doped bioglass nanoparticles were produced using a quick alkali-mediated sol-gel methodology optimized by our group for the production of BGs,${ }^{[19]}$ which was modified considering some procedures for synthesis of silver doped bioglass nanoparticles already reported in literature. ${ }^{[5,17,18]}$ Then, this previous sol-gel methodology was adapted for the production of bioglass nanoparticles with medium silver content: $\left[\mathrm{SiO}_{2}-\mathrm{CaO}-\mathrm{P}_{2} \mathrm{O}_{5}-\mathrm{Ag}_{2} \mathrm{O}\right.$ (mol\%):56-30-4-10] to maximize their antibacterial behavior and also to explore the effect of the Ag addition on their in vitro bioactivity. Firstly, to prepare the acidic solution (solution A), ethanol absolute was added to osmotized water, the $\mathrm{pH}$ value of this mixture was adjusted to 2 with nitric acid (2M) and, after $1 \mathrm{~h}$, tetraethyl orthosilicate (TEOS, $\left.\mathrm{Si}\left(\mathrm{OC}_{2} \mathrm{H}_{5}\right)_{4}\right)$ was added. This solution A was maintained under stirring during $1 \mathrm{~h}$ for the acidic hydrolysis of the precursor of $\mathrm{SiO}_{2}\left[\mathrm{Si}\left(\mathrm{OC}_{2} \mathrm{H}_{5}\right)_{4}\right]$. Then, for producing the solution $\mathrm{B}$, calcium nitrate tetrahydrate, ammonium phosphate dibasic and silver nitrate were sequentially added to 
osmotized water and the $\mathrm{pH}$ value was adjusted to 11.5 with ammonium hydroxide solution.

This reaction mixture was kept under stirring for $1 \mathrm{~h}$ to the basic hydrolysis of those precursors of $\left.\mathrm{CaO}\left[\mathrm{Ca}\left(\mathrm{NO}_{3}\right)_{2} .4 \mathrm{H}_{2} \mathrm{O}\right)\right], \mathrm{P}_{2} \mathrm{O}_{5}\left[\left(\mathrm{NH}_{4}\right)_{2} \mathrm{HPO}_{4}\right]$ and $\mathrm{Ag}_{2} \mathrm{O}\left[\mathrm{AgNO}_{3}\right]$.

Under smooth stirring, the solution A was slowly added to the solution B drop-by-drop and, during this step, the $\mathrm{pH}$ value of solution $\mathrm{B}$ was maintained at 11.5 using ammonia hydroxide solution. Then, the final solution was kept under stirring for $48 \mathrm{~h}$ in order to complete the condensation reaction that leads to gelation of bioglass. After this period, the stirring was stopped for precipitation of the gel particles, the supernatant was carefully removed and the precipitated gel was washed with osmotized water, at least three times. Finally, based on the thermal treatment described by Delben et al., ${ }^{[17]}$ after drying the obtained gel at $130^{\circ} \mathrm{C}$ for 24 $\mathrm{h}$ in an oven to evaporate the alcohol and water, and the obtained xerogel was treated at $600^{\circ} \mathrm{C}$ for $4 \mathrm{~h}$ to eliminate organic solvents, obtaining the $\mathrm{AgBG}$ powder.

\subsubsection{Characterization of $A g B G s$}

The Zeta potential, $\zeta$, was determined with $0.06 \%$ (w/v) AgBGs suspensions prepared with $0.15 \mathrm{M} \mathrm{NaCl}$ solution under magnetic stirring for $30 \mathrm{~min}$, and further ultrasonic dispersion at $25^{\circ} \mathrm{C}$ during $15 \mathrm{~min}$. The Zeta potential of the nanoparticles was measured three times by a Zetasizer equipment (Nano-ZS) from Malvern Instruments with a Dispersion Technology Software (DTS).

The morphology and composition of the glass powders were analyzed using SEM and the elemental composition of the bioglass powders was determined by EDS. X-ray diffraction (XRD) experiments were performed on a Bruker AXS D8 Discover with $\mathrm{Cu}-\mathrm{K} \alpha$ radiation $(\lambda=1.54060 \AA)$, scanning from $15^{\circ}$ to $60^{\circ}$ at a speed of $0.04^{\circ} \mathrm{s}^{-1}$. The analysis for phase identification was performed using analytical software EVA. The crystalline phases were indexed using the ICDD database (International Center for Diffraction Data). Fouriertransform infrared (FTIR) spectroscopy analysis was carried out in a PERKIN-ELMER 1600 FTIR spectrometer in Attenuated Total Reflection, ATR, mode as this technique is simpler to 
use than the conventional transmission mode. The sample preparation only required the scratching of glasses powders into the SiC abrasive sticks. The FTIR spectra were recorded from 400 to $4000 \mathrm{~cm}^{-1}$.

\subsubsection{In vitro bioactivity study}

Standard in vitro bioactivity studies were conducted immersing the synthesized AgBGs in a simulated body fluid, SBF, solution since it simulates the concentration of ionic species presents in human blood plasma. The SBF solution was prepared by following the Kokubo and Takadama protocol ${ }^{[21]}$ and its $\mathrm{pH}$ was adjusted to 7.4. Each glass coverslips with $10 \times 10$ $\mathrm{mm}^{2}$ was coated with carbon tape containing $\mathrm{AgBGs}$ and, then, they were immersed during 7 and 14 days in $25 \mathrm{~mL}$ of $\mathrm{SBF}$ solution at $37^{\circ} \mathrm{C}$. After removing from SBF solution, these glass pieces were cleaned with ultrapure water and dried at room temperature. The formation of apatite was characterized by Transform Infrared spectroscopy (FTIR) and X-ray diffraction (XRD) analysis.

\subsubsection{Microbiological analysis}

The antimicrobial properties of AgBGs nanoparticles were tested, in comparison with the ternary formulation (BGs), through the disk diffusion methodology. Briefly, circular glass coverslips were coated with few drops of $5 \mathrm{mg} \cdot \mathrm{mL}^{-1} \mathrm{AgBG}$ and BGs water suspensions, and then, they were placed on top of a Mueller-Hinton agar plate inoculated with $1.5 \times 10^{8} \mathrm{CFU}$ of a standardized culture of Staphylococcus aureus (+) and Escherichia coli (-), both with OD600 of approximately 0.1 , and incubated for $16 \mathrm{~h}$ at $37^{\circ} \mathrm{C}$. The formation of an inhibition zone surrounding the coated glass coverslips was used as indicator of antibacterial activity.

\section{Results and discussion}

Since the sol-gel methodology has been commonly used to synthesize silicate glass, we proposed a new procedure for AgBGs production through the modification of the sol-gel methodology developed by our group for ternary BGs synthesis. ${ }^{[19,20]}$ This previous sol-gel procedure involves a two-step acid-base catalysis, where the mixture of an acidic solution 
(water+ethanol+Si $\left.\left(\mathrm{OC}_{2} \mathrm{H}_{5}\right)_{4}\right)$ catalyzed by citric acid with a basic solution (water $+\left(\mathrm{NH}_{4}\right) 2 \mathrm{HPO}_{4}+\mathrm{Ca}\left(\mathrm{NO}_{3}\right)_{2} .4 \mathrm{H}_{2} \mathrm{O}$ ) catalyzed by ammonia water, leads to the condensation (gelation) of the precursors. Alcohol and water were the byproducts of this condensation reaction, which evaporated during drying step forming a xerogel. ${ }^{[3,19]}$ Based on the procedure of El-Kady et al., ${ }^{[5]}$ we only replaced citric acid by nitric acid as the catalyst of the acidic solution (solution A) and, comparing with the previous procedure for the ternary formulation, ${ }^{[19]}$ it increased the rate of condensations reactions and reduced the gelation time. Firstly, the zeta potential of the silver doped bioglass nanoparticles was investigated in a physiological saline medium ( $0.15 \mathrm{M} \mathrm{NaCl}$ solution) and the produced $\mathrm{AgBG}$ sresented a potential value of $-22.9 \pm 0.1 \mathrm{mV}$ at $\mathrm{pH} 5.5$ and, in comparison with the zeta potential determined for ternary bioactive nanoparticles $(-15.7 \pm 0.5 \mathrm{mV})$, this result indicated that silver doped nanoparticles have stability and they could better resist to aggregation. ${ }^{[22]}$ It is mostly known that a negative zeta potential is crucial to promote the bioactive behavior, ${ }^{[23]}$ since negative surfaces enhance the stable formation of surface apatite. So, it can be concluded that the developed silver doped nanoparticles presented an adequate zeta potential for the envisaged application.
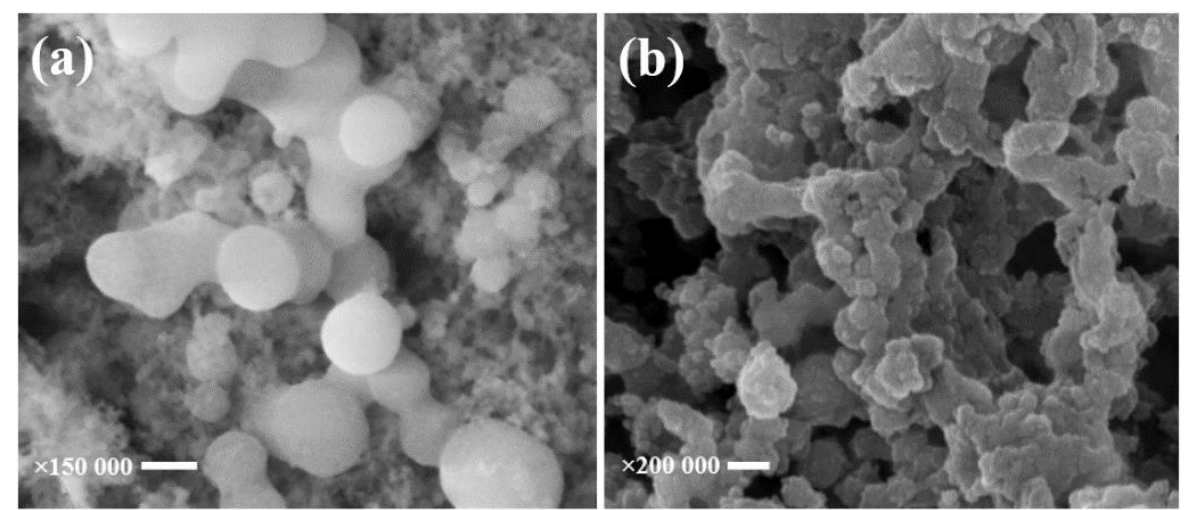

Fig. 1. Representative SEM images of the AgBGs (a) before and (b) after thermal treatment.

Fig. 1(a) shows the SEM image of the gel phase (xerogel) of AgBGs, while Fig. 1(b) shows the SEM image of AgBGs after calcination at $600^{\circ} \mathrm{C}$. SEM images revealed that the grain size of samples was less than $200 \mathrm{~nm}$. It can be seen that the morphology of the nanoparticles is 
more spherical and presents less agglomeration on the xerogel, which probably indicates that thermal treatment promotes the agglomeration of AgBGs, changing their morphology and reducing their final grain size.

Through the EDS, it was possible to quantify the final ratios of silver doped bioactive nanoparticles in terms of weight percentage, $\mathrm{Wt}(\%)$, after and before the calcination step. The EDS results are shown in Table $\mathbf{1}$ and the quantitative analysis presents deviations regarding the initial formulations: only $\mathrm{Si}$ is at the original percentage and $\mathrm{P}$ is five times higher, and consequently, $\mathrm{Ca}$ is $15 \%$ below the original percentage.

Table 1. Quantitative EDS analysis for the main precursors of AgBGs sol-gel and AgBGs after thermal treatment.

\begin{tabular}{lll} 
Element & $\begin{array}{l}\mathrm{AgBG} \text { Sol-gel } \\
\mathrm{Wt}(\%)\end{array}$ & $\begin{array}{l}\mathrm{AgBG}\left(130^{\circ} \mathrm{C}+600^{\circ} \mathrm{C}\right) \\
\mathrm{Wt}(\%)\end{array}$ \\
\hline $\mathrm{Si}$ & 21.7 & 16.3 \\
$\mathrm{Ca}$ & 9.7 & 12.5 \\
$\mathrm{Ag}$ & 2.1 & 1.9 \\
$\mathrm{P}$ & 1.6 & 1.5 \\
\hline
\end{tabular}

The reasons for these differences may be the removal of free calcium ions during the washing step. Indeed, in the sol stage, almost $100 \%$ of Ca dissolves in the pore liquor as calcium nitrate, and it will only deposit in the drying step. Therefore, the final composition of AgBGs can be changed if the pore liquor is washed before drying, removing the calcium that was not incorporated in the silica or phosphoric gel network. This fact could be related with the variation in values obtained, as the experimental procedure followed in this work included a washing step, which is crucial to remove the ammonia from the precipitate. Nevertheless, the EDS analysis confirmed that the produced nanoparticles contained silver. It can be seen that the analyzed samples presented silica, calcium and phosphorous compositions that were typically detected for any silicate bioglass ${ }^{[12,22]}$ and, also as expected, the silver content. Therefore, the sol-gel procedure proposed in the present work allowed a successful incorporation of silver ions in the composition of the bioglass nanoparticles. 
Fig. 2 shows the XRD pattern of AgBGs before (0 days) and after SBF immersion ( 7 and 14 days).

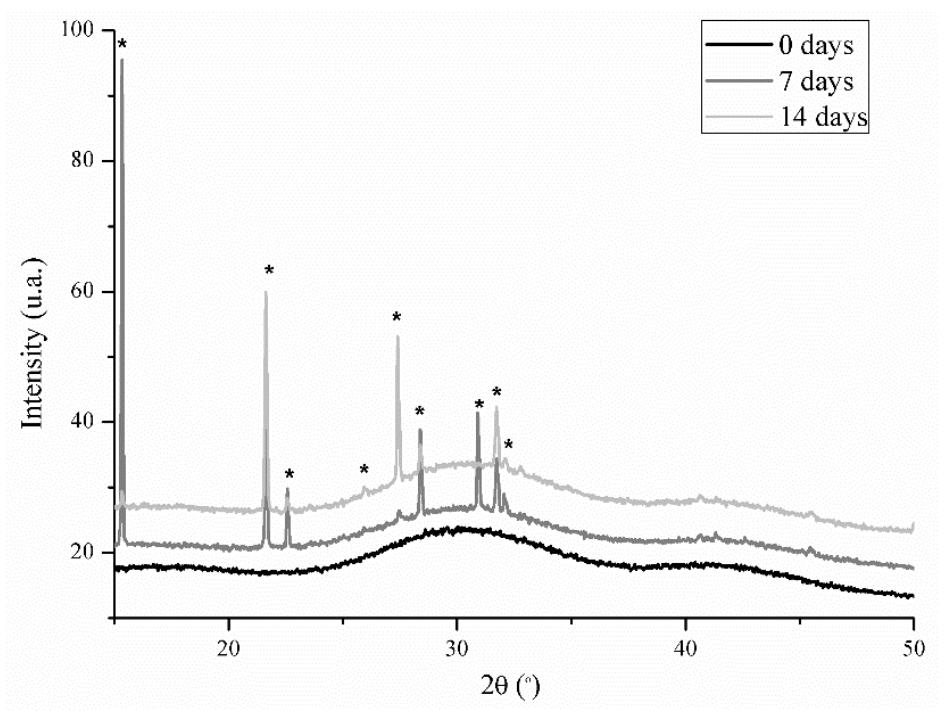

Fig. 2. XRD diffractograms of AgBGs obtained before and after the immersion in SBF for 7 and 14 days. Typical crystalline peaks of apatite-like phase were indicated.

Before SBF immersion, as expected, XRD diffractrogram presents an amorphous profile, only presenting the crystalline phases of calcium silicate and phosphorus oxide.

In fact, it is well known that glass is an amorphous inorganic material with no detectable diffraction maxima, but it seems that the thermal treatment process leads to the typical crystalline peaks of apatite-like phase (tricalcium phosphate). ${ }^{[19]}$ On the other hand, the precipitation of tricalcium phosphate can occur from the amorphous structure of silica-based bioglasses during the sol-gel process in the aqueous environment. ${ }^{[16]}$ After SBF immersion, some bands tend to disappear due to the decrease of Si percentage after SBF immersion, but all spectra revealed some typical crystalline peaks of apatite-like phase indicated at $2 \theta=15$ to $35^{\circ}$, as previously reported in other works. ${ }^{[7,17-19,24]}$ Attending these results, it was possible to conclude that these AgBGs presented in vitro bioactive character. Moreover, in accordance with El-Kady et al. ${ }^{[5]}$ and Vulpoi et al., ${ }^{[18]}$ our results suggest that the incorporation of a medium amount of silver into the bioglass system does not affect its bioactivity behavior. The FTIR spectra of AgBGs, after and before SBF immersion, were shown in Fig. 3. 


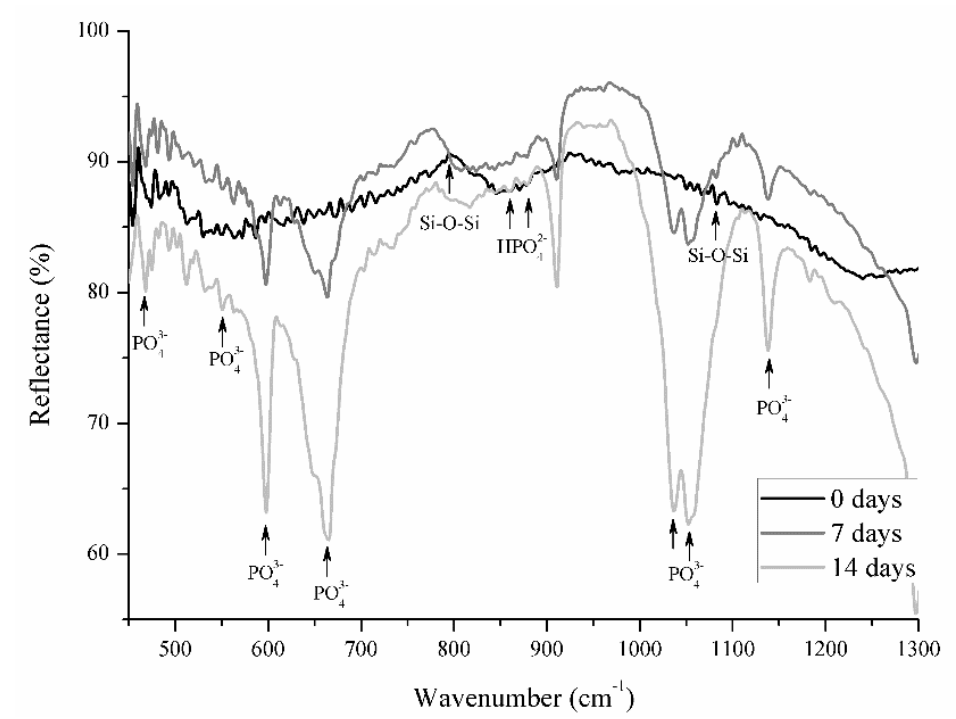

Fig. 3. FTIR spectra of AgBGs obtained before and after the immersion in SBF for 7 and 14 days.

After SBF immersion, it can be seen the band corresponding to Si-O-Si (1000-1200 $\left.\mathrm{cm}^{-1}\right)$ asymmetric stretching vibration, the one attributed to the $\mathrm{Si}-\mathrm{O}-\mathrm{S}\left(725-800 \mathrm{~cm}^{-1}\right)$ symmetric stretching vibration, and another one corresponding to the Si-O-Si $\left(450-480 \mathrm{~cm}^{-1}\right)$ bending mode, similarly with some characterization already reported. ${ }^{[5,16,18]}$

The bands represented in Fig. 3 are the typical ones in literature concerning bioglass nanoparticles, ${ }^{[5,19]}$ which revealed the formation of crystalline apatite-like phase confirming the bioactive character of the produced AgBGs. In fact, Luz et al. ${ }^{[19]}$ reported similar bands with ternary BGs and El-Kady et al. ${ }^{[5]}$ also showed that AgBGs presents the similar bands of those ternary system nanoparticles.

Finally, the antimicrobial properties of the synthesized nanoparticles were also investigated.

Fig. 4 shows representative images of the results obtained from the antibacterial tests performed with AgBGs and BGs (as control). Based on the region of inhibition observed in these images, even after 16h, the AgBGs clearly evidenced an antibacterial effect against S.aureus and E.coli, in accordance with the findings reported in some previous works, where silver nanoparticles with similar formulations were also characterized. ${ }^{[5,9,16]}$ 


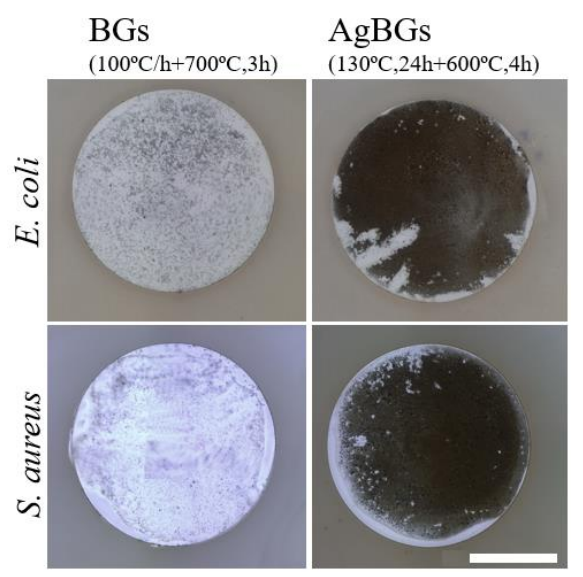

Fig. 4. Images of glass coverslips coated with BGs and AgBGs, 16 h after being placed on top of a Mueller-Hinton agar plate with S.aureus(+) and E.coli(-) (1.5x108 CFU) incubated at $37^{\circ} \mathrm{C}$. Scale bar represents $8000 \mu \mathrm{m}$.

Since ternary nanoparticles did not present microbial growth inhibition, it can be concluded that the addition of silver on the AgBGs provided their antimicrobial behavior. In the present study, it was tested bioglass nanoparticles doped with a medium silver content (10\% mol), in accordance with Chatzistavrou et al. ${ }^{[13]}$ that already reported the bactericidal action of silver nanoparticles, even for formulations with a lower concentration of $\mathrm{Ag}$. Bellantone et al ${ }^{[9]}$ also investigated the effect of $\mathrm{AgBGs}$ with different $\mathrm{Ag}_{2} \mathrm{O}$ contents on $S$. aureus and E. coli and they suggested that the antibacterial action of silver nanoparticles can be attributed to the leaching out of silver ions from the glass structure. Moreover, this antibacterial effect was also reported by Feng et al. and Kim et al., ${ }^{[8]}$ which suggested that silver ions dissolved out in the medium could react with bacterial metabolism, inhibiting its growth.

\section{Conclusions}

New silver doped bioglass nanoparticles $\left[\mathrm{SiO}_{2}-\mathrm{CaO}-\mathrm{P}_{2} \mathrm{O}_{5}-\mathrm{Ag}_{2} \mathrm{O}\right.$ (mol\%):56-30-4-10] were successfully prepared, following an alkali-mediated sol-gel methodology. Their Zeta potential values proved their stability and also their bioactive behavior. SEM analysis evidenced the production of nanosized particles and EDS quantitative analysis confirmed the successful incorporation of the silver on these bioglass nanoparticles. XRD patterns showed the crystalline peaks of apatite-like phase and FTIR spectra showed characteristic peaks of the siloxane bonds formed during the condensation reaction of silicate glasses. The 
microbiological tests revealed that the produced $\mathrm{AgBG}$ had an antibacterial effect against two different types of bacteria, which suggest their potential ability to inhibit bone infections.

Summarizing, the proposed sol-gel procedure provide a quick and effective production of nanosized and antibacterial AgBGs with medium content of silver, without compromising their in vitro bioactivity behavior. The synthesized silver doped nanoparticles revealed promising properties that could be implemented in several orthopedic applications, since the combination of bioactive with antibacterial properties is crucial to reduce the risk of postsurgery complications, mostly caused by severe bacterial infections upon implantation.

Received: ((will be filled in by the editorial staff))

Revised: ((will be filled in by the editorial staff)) Published online: ((will be filled in by the editorial staff))

[1] N. M. Alves, I. B. Leonor, H. S. Azevedo, R. L. Reis, J. F. Mano, J. Mater. Chem. 2010, 20, 2911.

[2] M. De, P. S. Ghosh, V. M. Rotello, Adv. Mater. 2008, 20, 4225.

[3] L. L. Hench, Curr. Opin. Solid State Mater. Sci. 1997, 2, 604.

[4] L. L. Hench, J. Mater. Sci. Mater. Med. 2006, 17, 967.

[5] A. M. El-Kady, A. F. Ali, R. A. Rizk, M. M. Ahmed, Ceram. Int. 2012, 38, 177.

[6] S. Silver, L. T. Phung, Ann. Rev. Microbiol. 1996, 50, 753.

[7] M. Shirkhanzadeh, M. Azadegan, J. Mater. Sci. Mater. Med. 1998, 9, 385.

[8] T. N. Kim, Q. L. Feng, J. O. Kim, J. Wu, H. Wang, G. C. Chen, F. Z. Cui, J. Mater. Sci. Mater. Med. 1998, 9, 129.

[9] M. Bellantone, N. J. Coleman, L. L. Hench, J. Biomed. Mater. Res. 2000, 51, 484.

[10] J. H. Crabtree, R. J. Burchette, R A. Siddiqi, I. T. Huen, L. L. Hadnott, A. Fishman, Perit. Dial. Int.: J. Int. Soc. Perit. Dial. 2003, 23, 368.

[11] S. A. Jones, P. G. Bowler, M. Walker, D. Parsons, Wound Repair Regen. 2004, 12, 288.

[12] B. S. Liu, T. B. Huang, Macromol. Biosci. 2008, 8, 932.

[13] X. Chatzistavrou, J. C. Fenno, D. Faulk, S. Badylak, T. Kasuga, A. R. Boccaccini, P. Papagerakis, Acta Biomater. 2014, 10, 3723.

[14] E. Vernè, S. D. Nunzio, M. Bosetti, P. Appendino, C. Vitale Brovarone, G. Maina, M. Cannas, Biomaterials 2005, 26, 5111.

[15] Q. L. Feng, J. Wu, G. Q. Chen, F. Z. Cui, T. N. Kim, J. O. Kim, J. Biomed. Mater. Res. 2000, 52, 662.

[16] M. Bellantone, N. J. Coleman, L. L. Hench, Key Eng. Mater. 2001, 192, 597.

[17] J. R. J. Delben, O. M. Pimentel, M. B. Coelho, P. D. Candelorio, L. N. Furini, F. Alencar dos Santos, F. S. de Vicente, A. A. S. T. Delben, J. Therm. Anal. Calorim. 2009, 97, 433.

[18] A. Vulpoi, L. Baia, S. Simon, V. Simon, Mater. Sci. Eng. 2012, 32, 178.

[19] G. M. Luz, J. F. Mano, Nanotechnology 2011, 22, 494014.

[20] G. M. Luz, J. F. Mano, J. Nanopart. Res. 2013, 15, 1.

[21] T. Kokubo, H. Takadama, Biomaterials 2006, 27, 2907. 
[22] A. Doostmohammadi, A. Monshi, R. Salehi, M. H. Fathi, Z. Golniya, A. U. Daniels, Ceram. Int. 2011, 37, 2311.

[23] H. H. Lu, S. R. Pollack, P. Ducheyne, J. Biomed. Mater. Res. 2000, 51, 80.

[24] D. S. Couto, N. M. Alves, J. F. Mano, J. Nanosci. Nanotech. 2009, 9, 1741. 